\title{
Acoustical variation and contextual specificity of a gorilla vocalization: A case study
}

\author{
Luef Eva Maria, Pika Simone \\ Max Planck Institute for Ornithology, Eberhard-Gwinner Strasse, 82319 Seewiesen, Germany
}

Email address:

eluef@orn.mpg.de (E. M. Luef), spika@orn.mpg.de (S. Pika)

\section{To cite this article:}

Eva Maria Luef, Simone Pika. Acoustical Variation and Contextual Specificity of a Gorilla Vocalization: A Case Study. Psychology and Behavioral Sciences,. Vol. 3, No. 6, 2014, pp. 192-196. doi: 10.11648/j.pbs.20140306.12

\begin{abstract}
Western lowland gorillas frequently produce a vocalization known as the DOUBLE GRUNT. It occurs in a variety of different behavioral contexts but so far no acoustical differences have been described that would distinguish DOUBLE GRUNT morphology in the particular contexts. The present study tests the hypothesis if different acoustical features characterize DOUBLE GRUNTS in different behavioral contexts. We are able to demonstrate that one acoustical variation of DOUBLE GRUNTS is consistently used in one behavioral context. Context-specificity of this kind has been described for certain chimpanzee calls and represents an interesting research avenue to investigate how species with a genetically determined vocal repertoire can achieve some degree of vocal variability. Our findings indicate that gorillas' vocalizations also comprise calls that can be acoustically modified to be used for specific contexts.
\end{abstract}

Keywords: Double Grunt, Context-Specific Call, Acoustic Variation

\section{Introduction}

The vocal repertoire of primates is largely constrained by genetic determination, which precludes learning processes from impacting on vocalizations to a considerable degree [1, 2]. For instance, the vocal types which are produced by western lowland gorillas are similar to those of mountain gorillas $[3,4]$, and a great degree of vocal uniformity is also characteristic for different sub-species of chimpanzees [5-7] and orang-utans [8]. Species with vocal learning abilities, on the other hand, such as song birds, can add new and drop old vocal types, rendering their vocal repertoire particularly flexible [as, for instance, sedge warblers, see 9]. While some flexibility in the vocal domain has been suggested for certain primate species [see, e.g., 10, 11-13], in particular concerning food-associated vocalizations [14], the overall degree of vocal learning remains limited and de-novo acquisition of new vocal types is largely impossible [15].

A genetic basis of vocalizations offers advantages in terms of cognitive load reduction, that is cognitive resources need not be used for conscientious acquisition processes [see, e.g., 16]. Furthermore, mutual understanding of conspecifics is ensured if every individual uses the same vocal types. Changing ecological or social conditions, however, may necessitate changes in vocal behavior of a species and thus require adaptations to the vocal repertoire [17]. There are various ways how restrictions superimposed by a genetic basis of vocalizations can be circumvented to some degree without developing fully-fledged vocal learning abilities: 1 . Call use may be expanded, i.e. a call that has previously only been used in a particular behavioral context starts to be used in a different context as well [termed "contextual learning", see 16], 2. Call-combinations are produced which convey a new 'meaning', as for instance in Campbell monkeys [see 18], and 3. The acoustical structure of a particular call is modified so that the result is multiple variants of one vocal type. Examples of this include barks of chimpanzees, where the different acoustic variants are used for different behavioral contexts [19].

Modifiable call production is a rare phenomenon in primates [20]. Clark and colleagues [21] reported wild gibbons (Hylobates lar) to modify song structure in response to different predators. Gibbon song is rigidly structured by its genetic basis [22] and song variation in response to external factors is therefore an unusual occurrence. Concerning the great apes, Crockford and Boesch [19] showed that wild chimpanzees (Pan troglodytes verus) adapt the acoustic nature of innate calls depending on the context in which they are 
emitted, e.g. barks in hunting contexts differ from those produced when a predator was encountered. Similarly, in pant-hoot vocalizations geographic variation can be found, suggesting that social factors such as extra-group discrimination play a role in acoustic structuring of this species-typical vocalizations [23].

Whether gorillas produce acoustical variants of innate call types is, so far, unknown. We therefore studied the vocal communication of two groups of free-ranging Western lowland gorillas and focused on the so-called DOUBLE GRUNT vocalization, a presumed contact call, that is emitted in a variety of different behavioral contexts [3]. The DOUBLE GRUNT vocalization is a two-component call consisting of two grunts uttered in quick succession and is one of the most frequent vocalizations of both mountain and western lowland gorillas [4, 24]. Originally referred to as double belch [25], the DOUBLE GRUNT is most often given by adult males and in a variety of different behavioral contexts such as resting, feeding, moving, or engaging in social activities, such as play [26]. We therefore developed the hypothesis that there may be acoustical differences between the DOUBLE GRUNTS given in four behavioral contexts: resting, leaving, addressing youngsters, and addressing females.

\section{Methods}

\subsection{Data Collection}

Data were collected between July and September 2012 from two free-ranging, habituated groups of western lowland gorillas in the Nouabalé-Ndoki National Park in the Republic of Congo. The Mondika study site is located at the boundaries of the Central African Republic and the Republic of Congo [for more detailled information see 27]. Two gorilla groups were followed daily from 7am until 4:30 pm by EML or an assistant (sampling rule: behavior sampling; recording rule: continuous recording, see [28]). The groups consisted of eleven and thirteen individuals and were observed for approximately 450 and 150 hours, respectively. Our recordings were made with a Sony PCM-M10 digital recorder (equipped with a directional Rode M3 microphone) at a sampling rate of 44.1 Hertz and 16 bits accuracy. Recordings were transferred digitally onto a MacBook Pro (OS X 10.6.8) and quantitative analyses of calls were carried out using Praat software (version 5.3.32, Boersma \& Weenink, 2007). We used the following setting for measurements: Spectrogram settings: view range $=0.0$ to $3000 \mathrm{Hertz}$, window length $=0.05$ seconds, dynamic range $=50 \mathrm{~dB}$; Intensity settings: view range $=50-100 \mathrm{~dB}$.

We focused our data collection on only silverback males (leaders of their groups) during natural communicative interactions with their conspecifics. DOUBLE GRUNTS were recorded at an average distance of 7 to 15 meters from the individuals. DOUBLE GRUNTS were recorded when the individuals were stationary, i.e. no body or head movements occurred beside from a slight head tilt upwards during vocalizing. Both silverbacks were habituated to an excellent degree [see 29]. For each vocal recording, additional behavioral data were collected to characterize the context of the vocalization: 1. Direction of DOUBLE GRUNT vocalization (direction of head of vocalizing silverback), 2. Activity of vocalizing silverback following within 30 seconds of a vocalization or the vocal bout (i.e. repeated DOUBLE GRUNTS). The 30-second mark was chosen as silverbacks, after uttering a DOUBLE GRUNT, would not immediately start acting but rather linger for some time in their position, either lying, standing, or looking at addressees. When no action had started after 30 seconds, we discarded the DOUBLE GRUNT from our data as no behavioral classification could be made.

We recorded the usage of DOUBLE GRUNTS in four frequently occurring contexts, termed REST, LEAVE, FEMALE, and REPRIMAND by us. In the REST context the silverback laid down on the forest ground for a prolonged resting period (minimum of five minutes) either within 30 seconds of uttering a single DOUBLE GRUNT or after producing the last grunt of a DOUBLE GRUNT bout. In the LEAVE context the silverback started to travel (i.e. initiated group movement), within 30 seconds after having used a single DOUBLE GRUNT or the last grunt of a DOUBLE GRUNT bout. In the FEMALE context the silverback directed his DOUBLE GRUNT toward a female passing him on the ground, as indicated by directing his attention (head movement) to her for the duration of the whole vocal utterance. In the REPRIMAND context the silverback uttered a DOUBLE GRUNT in the direction of immature individuals (infants, juveniles, subadults; ages 2 to 6) "harassing" him during resting periods. Harassing took the form of physical disturbance by youngsters bumping into the silverback or falling onto him during resting periods. The silverback would emit a DOUBLE GRUNT in the direction of the immature individual immediately following the harassment (within 3 to 5 seconds).

The study was approved by the Wildlife Conservation Society (Ref. Nr. 084/ DV/ WCS-12) and authorized by the Congolese Ministère de la Recherche Scientifique (Délégation Générale à la Recherche Scientifique et Technologique, Ref. Nr. 009/ MRS/ DGRST/ DMAST).

\subsection{Data Analysis}

For each silverback male, first the frequency of double grunting was calculated (DOUBLE GRUNTS per hour). For acoustical analyses, DOUBLE GRUNT recordings were uploaded onto Praat and analyzed. For each DOUBLE GRUNT, we measured the following temporal acoustic parameters: 1. The length of first grunt (in milli-seconds); 2. The length of second grunt (in milli-seconds); 3. The length of pause between the two grunts (in milli-seconds); 4. The overall length of the DOUBLE GRUNT vocalization (length of both grunts plus length of pause in between them). Temporal acoustic features, such as duration, are more easily modified than frequency parameters [17] and we thus focused our analysis on temporal measurements of DOUBLE GRUNTS. 


\section{Results}

Frequency of DOUBLE GRUNTS in the two silverback males yielded differences (silverback 1: $\mathrm{N}=179$, silverback 2: $\mathrm{N}=5$ ). Silverback 1 gave 81 DOUBLE GRUNTS in the REST context, 51 in the LEAVE context, directed 22 DOUBLE GRUNTS at females that were approaching him (FEMALE context), and gave 25 DOUBLE GRUNTS when addressing younger animals that were harassing him during resting periods (REPRIMAND context). Silverback 1 vocalized at a frequency of 0.4 DOUBLE GRUNTS per hour, while silverback 2 emitted 0.03 DOUBLE GRUNTS per hour. The subsequent analysis is thus limited to DOUBLE GRUNTS of silverback 1 .

Prior to the analyses, two variables (duration second grunt, overall length) were subjected to a logarithmic transformation (including zero values) in order to create a normal distribution in the measurements [see e.g. 30]. A One-Way ANOVA showed that three parameters of DOUBLE GRUNTS differed significantly between contexts: 1 . duration first grunt $\left(F=2.931, \mathrm{df}=3, \eta^{2}=0.72, \mathrm{p}=0.035\right), 2$. duration second grunt $\left(F=69.901, \mathrm{df}=3, \eta^{2}=0.81, p=0.000\right), 3$. overall length $\left(F=49.635, \mathrm{df}=3, \eta^{2}=0.89, p=0.000\right)$. The parameter duration pause fell short of statistical significance $(p=0.1)$. Post-hoc tests (Tukey-HSD) revealed that duration first grunt nearly reached significance between the two contexts REST and REPRIMAND $(p=0.051)$, duration second grunt yielded significant differences between the FEMALE context and all other contexts $(p<0.001)$, and overall length parameter showed significant differences between DOUBLE GRUNTS in the FEMALE and all other contexts $(p<0.001)$.

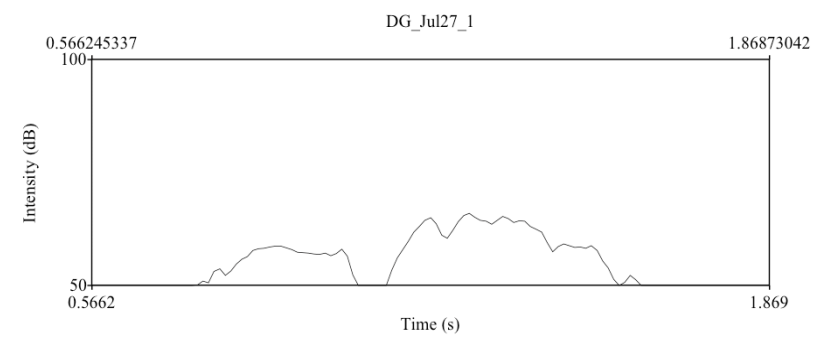

Figure 1. Length of a DOUBLE GRUNT given during resting

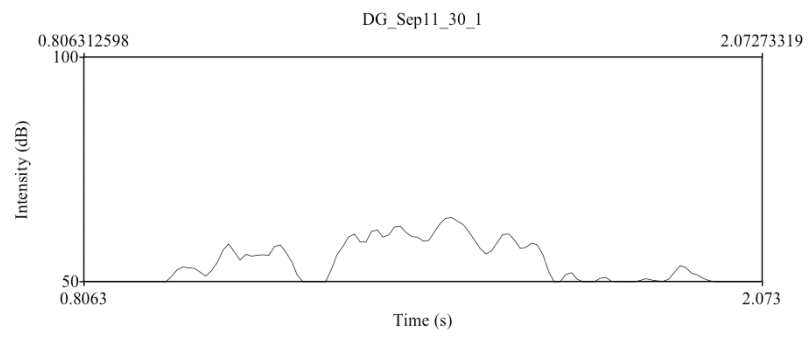

Figure 2. Length of a DOUBLE GRUNT directed at a female

We proceeded to conduct a discriminant function analysis (DFA) to further analyze the data. DFA probes data to determine relationships among several identified groups and assesses the discriminability between those groups based on measured values of the variables [31]. DFA is used to determine which continuous variables discriminate between two or more naturally occurring groups. The analysis operates via a two-step process: 1 . Testing significance of a set of discriminant functions, and 2. Classification [32]. Before running the DFA, we checked for outliers of the data and selected the individual parameters for the analysis (length of first and second grunt, length of pause, overall length). Wilk's $\lambda$ statistics was used to determine the contribution of each variable to the ability of DFA to classify contexts. The DFA yielded a significant discriminant function concerning one parameter, duration of second grunt: Wilk's $\lambda=0.455, \chi^{2}(3)=$ $69.901, p=0.000$. The eigenvalue $(=1.198)$ and the canonical correlation $(0.738)$ indicate that the function can discriminate the context well. The discriminant function correctly reclassified DOUBLE GRUNTS in $85.2 \%$ of cases for the context REST and in $63.6 \%$ of cases in the context FEMALE. The DFA could not distinguish between the majority of DOUBLE GRUNTS from the contexts REST, LEAVE, and REPRIMAND, and reclassified them into one group (REST). This shows that significant results from the previously conducted ANOVA could not hold under tighter scrutiny of the DFA. DOUBLE GRUNTS from the context FEMALE, however, were discriminated from the other contexts (see figures 1 and 2). An exact 2-tailed binomial test demonstrated that the cross-validated classification was still significantly higher than expected by chance (chance level $=0.25$, FEMALE: $p=0.012$ ).

\section{Discussion}

The aim of the present study was to investigate whether DOUBLE GRUNTS in wild gorillas show acoustical variation according to behavioral context. We found evidence that DOUBLE GRUNTS emitted in one context (FEMALE) are markedly different from those emitted in three other contexts (LEAVE, REST, REPRIMAND). DOUBLE GRUNTS given in the FEMALE context had significantly longer second elements. Lengths of a vocalization may be related to the distance of the recipient of the vocalization and to the surrounding forest habitat [33, 34], where prolonging of the vocalization may facilitate perceptibility and thus ensure reception [see 35]. During resting and leaving periods, however, the silverback was usually at a greater distance to the other group members than in REPRIMAND or FEMALE contexts. The distinguished DOUBLE GRUNT directed at females may serve a special communicative function between females and silverbacks that is related to communicative content rather than external environmental factors. We never observed a particular reaction of the females to the DOUBLE GRUNTS but they generally just walked by the silverback, sometimes looking at him while passing (personal observation).

The results of our study are largely congruent with a previous investigation of the acoustical correlates of DOUBLE GRUNTS in mountain gorillas [24]. Seyfarth and colleagues could not find differences in acoustical structure of DOUBLE GRUNTS in the contexts feed, rest, leave, and social behaviour. Our study included the contexts REST and 
LEAVE, however, we did not include feed and we further sub-divided social behaviour into two distinct contexts, FEMALE and REPRIMAND. Our results also showed that there were no differences between the REST and LEAVE context (the two congruent parameters between the two studies). Seyfarth et al. (1994) did not investigate the context FEMALE (or do not mention it to be included in their "social" context), which our data has shown to be characterized by an acoustically distinct DOUBLE GRUNT variant.

An important consideration in gorilla vocal behavior is idiosyncratic variability. The two silverbacks may experience different motivational states for double grunting in general or for the acoustic alteration of DOUBLE GRUNTS. Our study may not be representative of western gorillas but may show a facet of the communicative abilities of one silverback male. Although being based on a relatively small sample size, our data also show that DOUBLE GRUNT production can be markedly different between two silverback males. The large idiosyncratic differences in double grunting may, to some extent, be related to the social make-up of the gorilla group. There can be great variability in group compositions of wild gorillas, largely owed to the fact that female as well as male dispersal may take place [see 36] and this may also have an impact on communicative behavior.

In sum, our results demonstrate that one silverback male distinguished between DOUBLE GRUNTS given in the context FEMALE and those emitted in other contexts. By prolonging the second grunt element, the silverback male was able to modify the DOUBLE GRUNT vocalization to such a degree that it led to consistent usage in only one context. Similar findings have been reported for a chimpanzee that produced different acoustical variants of food grunts in relation to the presentation of different food items [12]. Acoustical variability of call types has rarely been reported in great apes and future studies are urgently needed to determine the role of call variation in the communication of primates in general and great apes in particular.

The gorilla DOUBLE GRUNT seems to be a complex vocalization that could potentially include more information than is currently assumed. Further research is needed to help clarify what factors influence DOUBLE GRUNT morphology and function in order to contribute to a more general understanding of this important gorilla vocalization.

\section{Acknowledgements}

This work was funded by a Fellowship of the Fritz-Thyssen Foundation (Germany) awarded to E. M. L. and a Sofja-Kovalevskaja-Award of the Alexander-von-Humboldt-Foundation (Germany) awarded to S. P. We thank the Ministère de la Recherche Scientifique of the Republic of Congo and the Wildlife Conservation Society for permitting us to work at Mondika.

\section{References}

[1] Zuberbuehler, K. (2012). Communication Strategies. In The evolution of primate societies, J.C. Mitani, J. Call, P.M. Kappeler, R.A. Palombit and J.B. Silk, eds. (Chicago and London: University of Chicago Press), pp. 643-663.

[2] Seyfarth, R.M., and Cheney, D.L. (1997). Some general features of vocal development in nonhuman primates. In Social influences on vocal development, C.T. Snowdon and M. Hausberger, eds. (Cambridge: Cambridge University Press), pp. 249-273.

[3] Harcourt, A.H., Stewart, K.J., and Hauser, M. (1993). Functions of wild gorillas 'close calls'. I. Repertoire, context, and interspecific comparison. Behaviour 124, 89-122.

[4] Salmi, R., Hammerschmidt, K., and Doran-Sheehy, D.M. (2013). Western gorilla vocal repertoire and contextual use of vocalizations. Ethol 119, 1-17.

[5] Crockford, C., and Boesch, C. (2005). Call combinations in wild chimpanzees. Behaviour 142, 397-421.

[6] Goodall, J. (1986). The chimpanzees of Gombe, (Cambridge, MA: The Belknap Press of Harvard University Press).

[7] Marler, P., and Tenaza, R.R. (1977). Signaling behavior of apes with special reference to vocalizations. In How animals communicate, T.A. Sebeok, ed. (Bloomington, IN: Indiana University Press), pp. 965-1033.

[8] Hardus, M.E., Lameira, A.R., Singleton, I., Morrogh-Bernard, H.C., Knott, C.D., Ancrenaz, M., Utami Atmoko, S.S., and Wich, S.A. (2009). A description of the orangutan's vocal and sound repertoire, with a focus on geographic variation. In Orangutans: Geographic variation in behavioral ecology and conservation, S.A. Wich, ed. (Oxford: Oxford University Press).

[9] Nicholson, J.S., Buchanan, K.L., Marshall, R.C., and Catchpole, C.K. (2007). Song sharing and repertoire size in the sedge warbler, Acrocephalus schoenobaenus: Changes within and between years. Animal Behaviour 74, 1585-1592.

[10] Fichtel, C., and van Schaik, C.P. (2006). Semantic differences in sifaka (Propithecus verreauxi) alarm calls: A reflection of genetic or cultural variants? . Ethology 112, 839-849.

[11] Crockford, C., Herbinger, I., Vigilant, L., and Boesch, C. (2004). Wild chimpanzees produce group-specific calls: A case for vocal learning? Ethology 110, 221-243.

[12] Slocombe, K.E., and Zuberbuhler, K. (2005). Functionally referential communication in a chimpanzee. Curr Biol 15, 1779-1784.

[13] Tanaka, T., Sugiura, H., and Nobuo, M. (2006). Cross-sectional and longitudinal studies of the development of group differences in acoustic features of coo calls in two groups of Japanese macaques. Ethology 112, 7-21.

[14] Clay, Z., Smith, C.L., and Blumstein, D.T. (2012). Food-associated vocalizations in mammals and birds: What do these calls really mean? Animal Behaviour 83, 323-330.

[15] Hammerschmidt, K., and Fischer, J. (2008). Constraints in primate vocal production. In The evolution of communicative creativity: From fixed signals to contextual flexibility, U. Griebel and K. Oller, eds. (Cambridge, MA: MIT Press). 
[16] Janik, V.M., and Slater, P.J.B. (2000). The different roles of social learning in vocal communication. Animal Behaviour 60, $1-11$.

[17] Janik, V.M., and Slater, P.J.B. (1997). Vocal learning in mammals. Advances in the Study of Behavior 26, 59-99.

[18] Arnold, K., and Zuberbuehler, K. (2008). Meaningful call combinations in a non-human primate. Current Biology 18, R202-R203.

[19] Crockford, C., and Boesch, C. (2003). Context-specific calls in wild chimpanzees, Pan troglodytes verus: analysis of barks. Anim Behav 66, 115-125.

[20] Seyfarth, R.M., and Cheney, D.L. (2010). Production, usage, and comprehension in animal vocalizations. Brain and Language 115, 92-100.

[21] Clarke, E., Reichard, U.H., and Zuberbuhler, K. (2006). The syntax and meaning of wild gibbon songs. PLoS One 1, e73.

[22] Geissmann, T. (2002). Duet-splitting and the evolution of gibbon songs. Biol Rev Camb Philos Soc 77, 57-76.

[23] Mitani, J.C., Hasegawa, T., Gros-Louis, J., Marler, P., and Byrne, R. (2005). Dialects in wild chimpanzees? . Am J Primatol 27, 233-243.

[24] Seyfarth, R.M., Cheney, D.L., Harcourt, A.H., and Stewart, K.J. (1994). The acoustic features of gorilla double grunts and their relation to behavior. Am J Primatol 33, 31-50.

[25] Fossey, D. (1972). Vocalizations of Mountain Gorilla (Gorilla-Gorilla-Beringei). Anim Behav 20, 36-53.

[26] Fossey, D. (1983). Gorillas in the mist, (Boston and New York: Mariner Books).

[27] Doran, D.M., McNeilage, A., Greer, D., Bocian, C., Mehlman,
P., and Shah, N. (2002). Western lowland gorilla diet and resource availability: New evidence, cross-site comparisons, and reflections on indirect sampling methods. Am J Primatol $58,91-116$.

[28] Martin, P., and Bateson, P. (1994). Measuring behaviour: An introductory guide, (London: Cambridge University Press).

[29] Doran-Sheehy, D.M., Derby, A.M., Greer, D., and Mongo, P. (2007). Habituation of western gorillas: the process and factors that influence it. Am J Primatol 69, 1354-1369.

[30] Lehner, P.N. (2002). Handbook of ethological methods, 2nd Edition, (Cambridge, UK: Cambridge University Press).

[31] Dillon, W.R., and Goldstein, M. (1984). Multivariate analysis, methods and applications, (New York: Wiley).

[32] Tabachnick, B.G., and Fidell, L.S. (1996). Using multivariate statistics, (New York: Harper Collins College Publisher).

[33] Mitani, J., Hunley, K.L., and Murdoch, M.E. (1999). Geographic variation in the calls of wild chimpanzees: A reassessment. Am J Primatol 47, 133-151.

[34] Wiley, R.H. (1994). Errors, exaggeration, and deception in animal communication. In Behavioral mechanisms in evolutionary ecology, L. Real, ed. (Chicago: University of Chicago Press), pp. 157-189.

[35] Wiley, R.H., and Richards, D. (1982). Adaptations for acoustic communication in birds: Sound transmission and signal detection. In Acoustic communication in birds, Volume 1, D.E. Kroodsma and E.H. Miller, eds. (New York: Academic Press), pp. 131-181.

[36] Robbins, M.M. (2007). Gorillas: Diversity in ecology and behavior In Primates in perspective, C.J. Campbell, A. Fuentes, K.C. MacKinnon, M. Panger and S.K. Bearder, eds. (New York: Oxford University Press). 